\title{
Scheduler Algorithm for IEEE802.11n Wireless LANs
}

\author{
Bakeel Maqhat, Mohd Dani Baba, Ruhani Ab Rahman, and Anwar Saif
}

\begin{abstract}
The IEEE 802.11n network main feature is to support high throughput of more than $100 \mathrm{Mbps}$ at the MAC layer. To achieve this, the 802.11n Task Group has introduced frame aggregation technique which reduces the overhead and increases the channel utilization efficiency. Some recent researches have enabled an A-MSDU selective retransmission which makes it robust against error. In this paper, we propose a scheduler based on an A-MSDU aggregation mechanism that supports small size and high sensitivity traffics, and enable prioritization according to the $\mathrm{QoS}$ requirements of the traffic classes.
\end{abstract}

Index Terms-802.11n, scheduling, QoS, A-MSD.

\section{INTRODUCTION}

With the increasing demands on real-time services in Wireless LAN systems, it becomes more essential to supply enhanced quality of service (QoS). In response to that, the IEEE 802.11 Task Group yielded the new standard named as IEEE 802.11e that functions to support the QoS real time services. Thus by supporting QoS and increasing the data rate that can ultimately lead to provide better QoS especially for multimedia applications. On the other hand, limitation arises because of the theoretical throughput limit. This happens due to the large MAC and PHY overhead [1].

To overcome this limitation and achieve a throughput of more than 100Mbps at the MAC layer, IEEE 802.11n task group has introduced many enhancements both at the PHY and MAC layer. The key enhancement in 802.11n MAC layer is the frame aggregation technique which combines multiple frames into one large aggregated frame before the transmission[2]. Combining multiple frames into one large frame increases the channel utilization and improves the MAC throughput. Furthermore, the frame aggregation plays a vital role in lowering the transmission time for multiple frame headers, preambles and further reduces the waiting time through carrier senses multiple access with collision avoidance (CSMA/CA) random back-off period for successive frame transmission [3].

The rest of this paper is organized as follows: Section II is the background and related work. In Section III we present the proposed scheduler scheme along with its algorithm. Section IV is the results and dissection. The conclusion is stated in Section V.

\section{BACKGROUND AND RELATED WORK}

The $802.11 \mathrm{n}$ standard introduces two frame aggregation schemes, aggregate MAC service data unit (A-MSDU) and aggregate MAC protocol data unit (A-MPDU). The first

Manuscript received September 9, 2013; revised February 2, 2014.

The authors are with Centre for Computer Engineering Studies, Faculty of Electrical Engineering, Universiti Teknologi MARA, ShahAlam, Malaysia (e-mail: Bakeel2007@yahoo.com, \{mdani074, ruhani467\}@salam.uitm.edu.my). scheme A-MSDU involves concatenating several MAC Service Data Units (A-MSDU) to create the data payload of MPDU. It functions at higher levels of the MAC layer. Two parameters are essential for creating A-MSDUs; the maximum length of an A-MSDU, 3839 or 7935 bytes by default, and the maximum waiting time before creating an A-MSDU. These MSDUs in one A-MSDU must be in the same traffic class (same traffic identifiers TID) with the same destination and source [2]. Fig. 1 shows the structure of the A-MSDU frame. Every frame consists of a subframe header, MSDU payload and variable padding (0-3bytes) to keep the subframe at a multiple of four bytes for deaggregation alignment on the receiver side. In this technique, any corruption frame will be excluded.

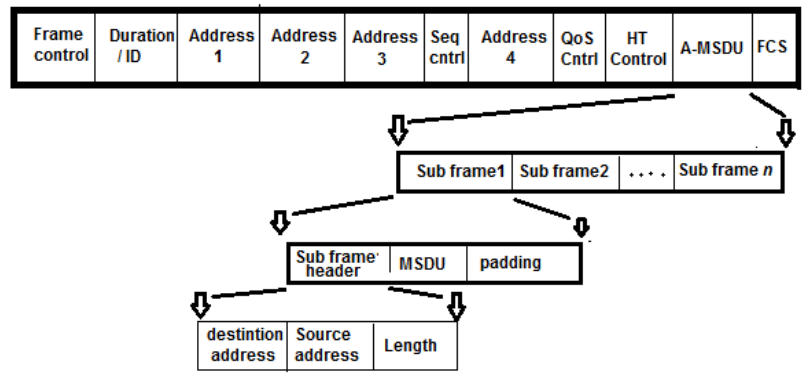

Fig. 1.The A-MSDU frame structure.

In the second scheme, several MPDUs are aggregated into one A-MPDU were performed on the lower part of the MAC layer. As A-MPDU comprises complete MPDUs, it can be aggregated as MPDUs of the different traffic class (different TID) and different Destination address. The corruption of any A-MPDU subframe does not require retransmission of the whole A-MPDU as only the corrupted ones are required for retransmission. The maximum size limit of A-MPDU is 65535 bytes. The structure of the AMPDU is shown in Fig. 2 where each frame consists of at least one complete MPDU subframe, and each subframe has delimiter and padding.

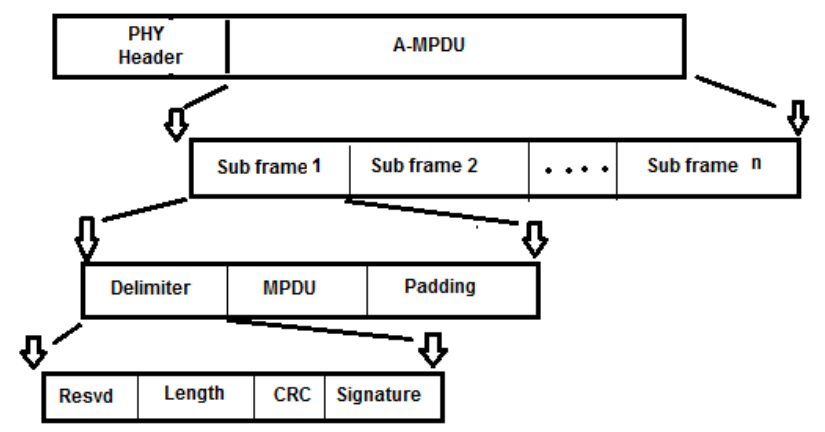

Fig. 2. The A-MPDU structure.

Some previous works as in [2], [4], [5] have investigated the frame aggregation schemes proposed in $802.11 \mathrm{n}$ standard. Skordoulis et al., [4] have revealed that the 
aggregation mechanisms primarily function to increase the channel efficiency and reduce the overhead. The study by Wang and Wei. [2], have concluded that aggregation scheme improves the channel efficiency and provides high quality of WLAN networking support for VOIP service due to its small frame size. Finally, the process of aggregating the small packets size applications like videos into one large frame can effectively improve the throughput performance [5].

The A-MPDU outperforms the A-MSDU due to the large size and the subframe retransmission capability in the erroneous channels; however, the A-MSDU outperforms the A- MPDU under error free channel and the same aggregation size due to its smaller aggregation headers [6]. Furthermore, the drastic usage of small size frames in the applications such as video, VoIP, interactive gaming as well as the large distribution of the small frames on the Internet, have created a pressing need to innovate the frame aggregation with compact headers and robustness against errors to meet better performing throughputs even under erroneous channels. To this end, several works have been proposed in the research literature to enable retransmission at the MSDU level, which contains small size headers.

Yu-Tzu. [7], proposed an aggregated selective repeat ARQ (ASRARQ) algorithm that utilizes the conventional selective repeat ARQ scheme which takes into account the frame aggregation. This algorithm will retransmit aggregated frames that have not been sent successfully until all the aggregated frames have either been transmitted successfully or reached the retry limit. Tianji et al., [8], introduced a new technique called aggregation with fragment retransmission (AFR). The function of this technique is to subdivide the received packets from the upper layer into small fragments, due to the uniqueness behavior of small frames by being less affected by the channel error in comparison to large ones. However, AFR scheme increases the header overhead with the number of fragments in the transmitted frame. Moreover, fragmentation andfragmentations processes require more processing time and large buffering. Saif. [9], introduced an MSDU frame aggregation called SRA-MSDU aggregation scheme to reduce the headers of the subframes and supports the retransmission of the corrupted MSDUs at the MSDU level. By incorporating control fields to the subframe header in order to enable a subframe integrity check and selective retransmission with the MAC header of A-MSDU frame being unchangeable as in Fig. 3. This algorithm results in improved system performance because of an increase throughput as well as decreasing the delay under various channel conditions.

As a result of non-existence of frame aggregation scheduling in the 802.11n standard; therefore it is left for the venders to interpret. The current $802.11 \mathrm{n}$ scheduler inherits the priority mechanism of the legacy 802.11e Enhanced Distributed Channel Access (EDCA) scheduler. EDCA scheduler defines several access categories (ACs). Each AC has a separate queue and different channel access waiting time depending on the ACs. Therefore, higher priority classes will lead the lower priority classes in channel access speed. By incorporating the EDCF scheduler to function with frame aggregation will results in low quality performance because of the small aggregation within the high priority access categories [10]. Therefore, new schedulers are needed to be implemented so as to satisfy the traffic requirements and co-work with the $802.11 \mathrm{n}$ aggregation features.

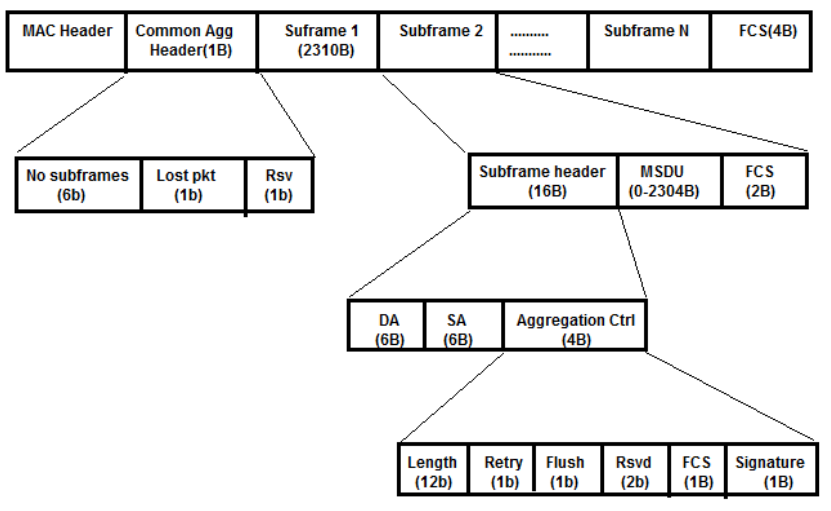

Fig. 3. The SRA-MSDU frame structure.

Yuxia, Lin et al., [3], studied the Bianchi model [11] to enhance frame aggregation schemes and suggested an optimal frame size adaptation algorithm for aggregation techniques under error prone channels. In ideal channel, [4] has examined the enhancement of throughput by using the aggregation schemes.

The studies by Haywood and Fallah [12]-[13], have tried to resolve the scheduling problem in WLAN by using contention windows, according to EDCF. On the other hand, H. Zen et al., [14], segregates real-time from non real-time traffic and transmitted in contention free period; however, this scheme requires substantial overhead for the transmission of polling frames from Access Point (AP) to the stations that transmitting real-time traffic. Ping and Weihua [15], have proposed a novel token-based scheme to eliminate collisions and subsequentlyincrease channel utilization. These authors have reported that, by integrating voice and data traffic, the token-based scheduling can leads to better result than DFC in terms of channel utilization. P. Koutsaki [16], has extended Weihua's work by the integration of video, voice and data traffic over WLAN. This scheme practically eliminates contention and transmission opportunity (TXOP) idle time which leads to increase in channel utilization when compared with EDCA. However, hidden node problems are not completely solved which would affect this scheme, as it uses token-passing.

An adaptive delayed channel access algorithm as proposed by Skordoulis et al., [17] to adapt channel access. This scheme introduces extra delay at MAC layer so as to increase the frame aggregation size, which in turn effects the QoS requirements of some ACs.

Pure opportunistic scheduling (POS) in wireless network provides the end users with the optimum channel conditions of transmission at a given time instant. Nonetheless, POS method maximizes the throughput; it may not succeed to do so when the frame aggregation is in place. In frame aggregation, the queues of the stations that are processed regularly will not cause the aggregation size to degrade. Small aggregation size will end up in low throughput even though high data rates are used.E. Ciftcioglu [18], have proposed an aggregate opportunistic scheduling (AQS) by 
combining aggregation and opportunistic scheduling approaches to enhance throughput and permits relatively fair access. However, it does not take into consideration the QoS requirements. Meanwhile F. Wang et al., [19], have introduced multi-user scheduling algorithm by taking the advantage of frame aggregation and adapt the knapsack problem so as to improve the system performance in terms of QoS efficiency. They use user urgency concept to schedule packets based on user's QoS requirements. The algorithm schedules the highest urgent frame that attribute to the highest urgent user. The urgency of user will decrease as soon as it gets serviced. However, the limitation of this scheduling algorithm arises from the anonymity of the initial user urgency and the dedication it exhibits to the downlink.

The frame aggregation scheduler proposed by Selvam and Srikanth [20], have crystallized the approach of aggregate frames by calculating the deadline based on the earliest expiry time of a frame waiting in the queue, and selecting dynamically the aggregation scheme based on frame aggregation size and bit error rate by using optimal frame size from the lookup table which proposed in [3]. However, this algorithm is restricted to deal with one type of traffic, thus other traffic will suffer from delay and eventually may affect the QoS requirements of other traffic.

Since some applications are time sensitive and the increase in demand of small frame size in applications such as video, audio, and interactive gaming, A-MSDU with compact headers become very suitable to enhance the system performance, especially after the improvement of head optimization and enabling frame retransmission, which make it robust against errors, [21] propose real-time scheduler by exploit the A-MSDU attributes. In this paper we propose scheduler works onan A-MSDU aggregation mechanism to supports small size and time sensitive traffic, and enable prioritization according to the QoS requirements of the traffic classes.

\section{THE PROPOSED SCHEDULER}

The proposed scheduling algorithm schedules the traffic which has time sensitive and different lifetimes. The work involves queuing the sender side calls the transmitting queue (TQ) and sorts the packet in ascending order based on thepriorities. According to that, packets which have highest priorities will occupy the top of the queue. The packet is taken from the top of TQ according to highest priorities and then insert at into the aggregated frame which is referred as the superframe.

The header of the received MSDUs from the upper layer has to be modified as suggested in [9] by adding control fields to the subframe header in order to enable retransmission and to create the MAC frame which in turn will be ready to aggregate. The received MSDUs from the upper layer are queued in the transmitting queue based on their priorities and arrival time. If there are two MSDUs have the same priority, the MSDU which arrived first is placed on the top of the transmission queue. Furthermore, while the construction of the aggregation frame, only the MSDUs that have the same destination address (DA) will be associated to the aggregation frame. The TQ sorting process is updated whenever a new MSDU is added to the TQ or an acknowledgment (ACK) bitmap is received. The aggregated frame is transmitted as soon as it reaches the aggregation size limit or the aggregation delay limit and $\left(T_{a g g}\right)$. The aggregation delay limit is estimated based on the Head of Queue packet (HoQP) arrival time. The absolute time to transmit an aggregated frame is the $T_{a g}$ without compromising the delay of the aggregated MSDUs. The $T_{a g}$ can be calculated by using the following equation:

$$
T_{a g g}=\frac{\left(T_{c r t}-H_{a r v}\right)}{T_{t x}}
$$

where:

$T_{a g g}:$ The aggregation delay limit

Harv: is the (HoQP) Head of Queue packet's arrival time.

$T_{\text {crt: }}$ represents the current time.

$T_{t x}$ : represents the required time to transmit the A-MSDU superframe.

$T_{t x}$ is updated as soon as a new MSDU is placed into the aggregated frame that is due to the increase of the existing size of the A-MSDU superframe. The notations used in this paper are listed in Table I.

The TQ will be updated once a Block Acknowledgement (BA) is received. When the considered MSDU is received correctly, it will be removed; otherwise it will be considered as corrupted. It will gain a high sending priority by placing at the top of TQ, and will be retransmitted at the head of the next aggregated superframe. The proposed scheduling algorithm for the time sensitive traffic is as shown in Fig. 4.

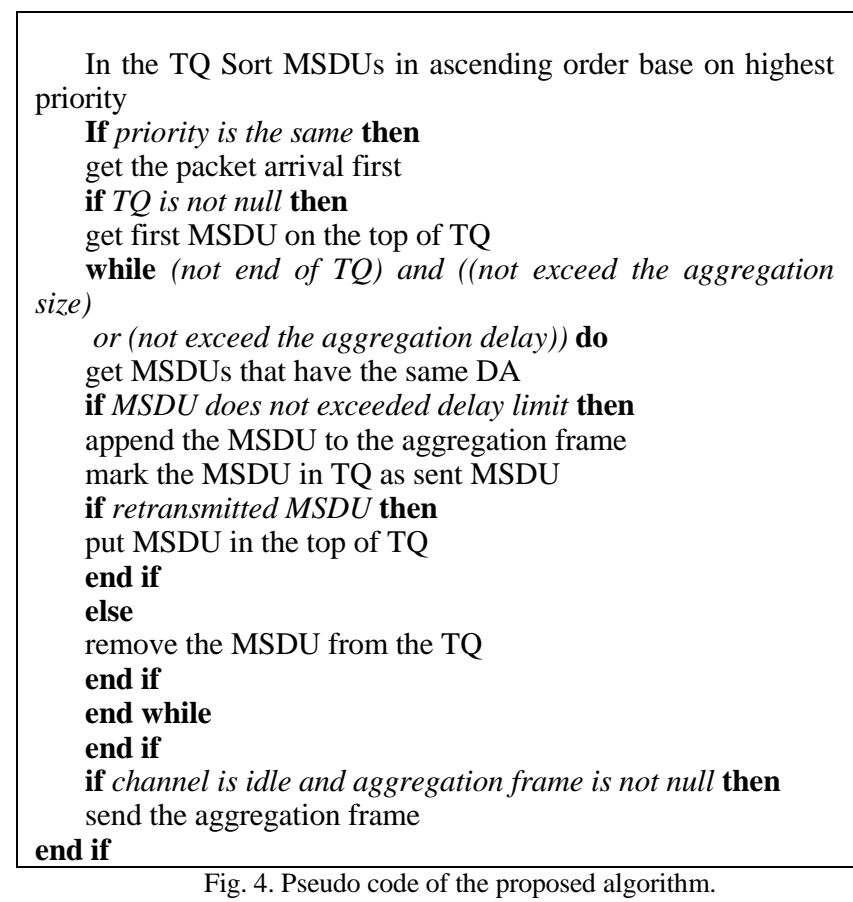

\section{RESUlt AND DisSECTION}

We carried out several simulation experiments using the network simulator (NS2) in order to evaluate the time sensitive traffics scheduling in terms of average delay and packet loss.Moreover, we have used the simulation scenarios number 17 of the point-to-point usage model [22]. This scenario comprises a fixed high throughput access 
point and station that are operating over a $20 \mathrm{MHz}$. Furthermore, the network traffic is composed of UDP flow based on constant bit rate (CBR) and the video conferencing with packet size of 512 bytes, Audio/Video streaming with packet size of 512 bytes and background traffic. Hence, the video rate is $2 \mathrm{Mbps}$ and the Audio/Video streaming rate is $1 \mathrm{Mbps}$ [22]. The characteristic of traffic is given in Table II.

TABLE I: NOTATIONS

\begin{tabular}{|l|l|}
\hline A-MSDU & MAC service data unit aggregation \\
\hline MSDU & MAC service data unit \\
\hline TQ & transmitting queue \\
\hline DA & destination address \\
\hline HoQP & Head of Queue packet \\
\hline $\mathrm{T}_{\mathrm{agg}}$ & aggregation delay limit \\
\hline Harv & HoQP arrival time \\
\hline Tcrt & current time \\
\hline $\mathrm{T}_{\mathrm{tx}}$ & transmit time \\
\hline
\end{tabular}

TABLE II: APPLICATION CHARACTERISTICS

\begin{tabular}{|l|l|l|l|}
\hline Application & Priority & Data Rate & Lifetime \\
\hline Video confrenceing & High & $2 \mathrm{Mbps}$ & $100 \mathrm{msec}$ \\
\hline A/V streaming & Medium & $1 \mathrm{Mbps}$ & $200 \mathrm{msec}$ \\
\hline
\end{tabular}

The Data rate is set to $300 \mathrm{Mbps}$ and basic rate is set to $54 \mathrm{Mbps}$. The average delay and packet loss ratio of the different traffic are examined under a different number of stations (i.e., varied from 10 to 60) with bit error rate (BER) set to $10^{-4}$. Other simulation parameters are listed in Table III.

In Fig. 5 and Fig. 6, we compare the average delay of the proposed scheduling scheme with the SRA-MSDU scheme. The proposed scheduling scheme achieves lower average delay than the existing scheme due to scheduling transmission mechanism, which relies on the type of traffic and their priorities. As well as sending different traffic in the single superframe rather than several superframes that will not experience a large delay due to TQ waiting time. From the graph we can see that the average delays will increase by increasing the number of stations due to the increasing in the contention time and superframes retransmission. Moreover, in the high erroneous channel the number of packets retransmits will increase further causing increasing in the average delay.

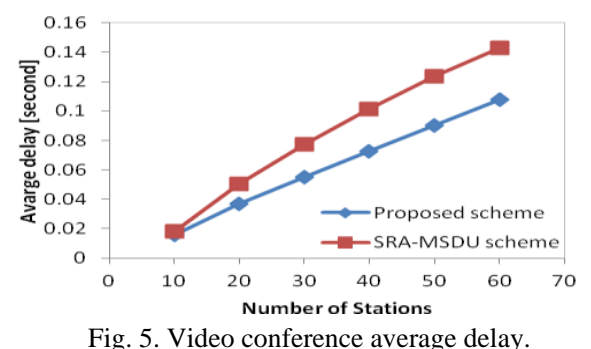

Fig. 7 and Fig. 8 show the difference in packet loss ratio between the proposed scheduling scheme and the SRAMSDU scheme. The packet loss ratio of the proposed scheduling scheme reaches about $50 \%$ as in Fig. 7 and reaches about $70 \%$ in Fig. 8. In both cases are lower than the packet loss ratio of the SAR-MSDU scheme. This is attributed to the proposed scheduling scheme, which guaranteed the packet to be served before its lifetime end. In addition, it gives the priority to the packet that has the lowest lifetime. Furthermore, we can see that from the figures, the packet loss ratio will increase by increasing the number of stations (i.e., which caused more traffic load) the high erroneous channel caused the packets transmission failure and increase the retransmit attempts.

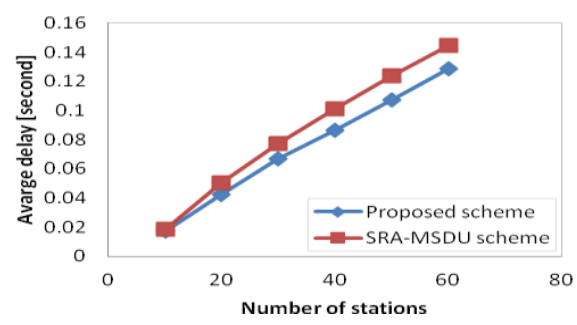

Fig. 6. A/V streaming average delay.

As shown in Fig. 9, the throughput of the proposed scheduler and SRA-MSDU with small number stations reaches about 65Mbps. In addition, the collisions will happen frequently in a high populated network and will affect the system performance. Therefore, when the number of contending stations is increasing the system throughput will decrease.

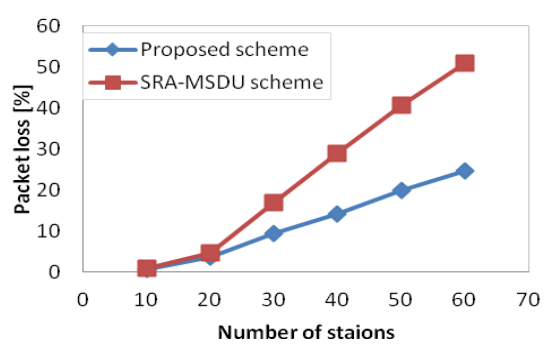

Fig. 7. Video conference packet loss ratio.

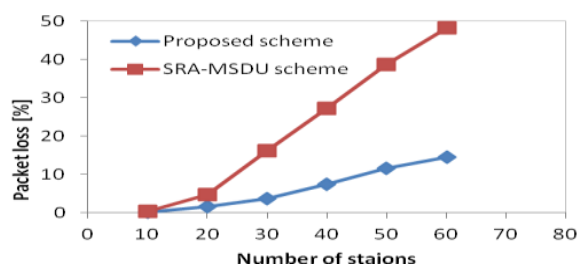

Fig. 8. A/V streaming packet loss ratio.

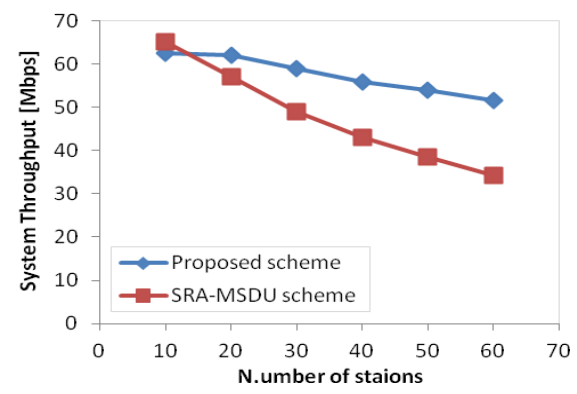

Fig. 9. System throughput.

TABLE III: SIMULATION PARAMETERS

\begin{tabular}{|l|l|}
\hline Parameter & Value \\
\hline TSIFS & $16 \mu \mathrm{s}$ \\
\hline Tphyhdr & $20 \mu \mathrm{s}$ \\
\hline Tidle & $9 \mu \mathrm{s}$ \\
\hline CWmin & 16 \\
\hline TDIFS & $34 \mu \mathrm{s}$ \\
\hline Data rate & $300 \mathrm{Mbps}$ \\
\hline Basic rate & $54 \mathrm{Mbps}$ \\
\hline
\end{tabular}

The throughput of the proposed scheduling scheme shows superior performance about $50 \%$ better than the SRAMSDU scheme by achieving high throughput whenever the 
number of stations is increased and the erroneous channel of 10e-4 s BER.

\section{CONCLUSION}

The current $802.11 \mathrm{n}$ standard does not specify the scheduling mechanism for frame aggregation but leave it to the interpretation of the vendors. In this paper we proposed different traffic classes scheduler that relies on traffic priority and takes into account the aggregation scheme which reduces the overhead in order to satisfy the QoS requirements.

In this algorithm, it primarily sorts the received traffic fromupper layer based on priorities in ascending order. According to that, packets which have highest priorities will occupy the top of the queue. The packet is taken from the top of TQ according to highest priorities and then insert at into the aggregated frame which is referred as the superframe.

Several simulations were conducted and the results show the superiority of the proposed scheduler scheme as compared to the SRA-MSDU scheme in terms of average delay and packet loss.

In the future we are planning to optimize the algorithm scheduler to be able contend with all traffic. Moreover, our algorithm can be enhanced to dynamically adapt to the network conditions based on traffic load measurement and erroneous channels.

\section{REFERENCES}

[1] L. Seungbeom and P. S. Chong, "Rotating priority queue based scheduling algorithm for IEEE 802.11n wlan," in Proc. the 9th International Conference on Advanced Communication Technology, 2007, pp. 1702-1706.

[2] C. Y. Wang and H. Y. Wei, "IEEE 802.11n mac enhancement and performance evaluation," Mobile Networks and Applications, vol. 14, pp. 760-771, 2009.

[3] L. Yuxia and V. W. S. Wong, "WSN01-1: frame aggregation and optimal frame size adaptation for IEEE 802.11n wlans," in Proc. Global Telecommunications Conference, 2006. GLOBECOM '06. IEEE, 2006, pp. 1-6.

[4] D. Skordoulis et al., "IEEE 802.11n MAC frame aggregation mechanisms for next-generation high-throughput WLANs," Wireless Communications, IEEE, vol. 15, pp. 40-47, 2008.

[5] Z. Haifeng et al., "Video transmission over IEEE 802.11n WLAN with adaptive aggregation scheme," in Proc. 2010 IEEE International Symposium on Broadband Multimedia Systems and Broadcasting (BMSB), 2010, pp. 1-5.

[6] K. Byung Soo et al., "Effect of frame aggregation on the throughput performance of IEEE 802.11n," in Proc. Wireless Communications and Networking Conference, 2008, pp. 1740-1744.

[7] H. Y. Tzu et al., "Performance analysis for aggregated selective repeat ARQ scheme in IEEE 802.11n networks," in Proc. 2009 IEEE 20th International Symposium on Personal, Indoor and Mobile Radio Communications, 2009, pp. 37-41.

[8] L. Tianji et al., "Aggregation With Fragment Retransmission for Very High-Speed WLANs," IEEE/ACM Transactions on Networking, vol. 17, pp. 591-604, 2009.

[9] A. Saif and M. Othman, "SRA-MSDU: Enhanced A-MSDU frame aggregation with selective retransmission in $802.11 \mathrm{n}$ wireless networks," Journal of Network and Computer Applications, vol. 36, no. 4, pp. 1219-1229, 2013.

[10] Y. Do et al., "Opportunistic scheduling in IEEE 802.11n WLAN," presented at the 6th Conference on WSEAS International Conference on Applied Computer Science, vol. 6, Hangzhou, China, 2007.

[11] G. Bianchi, "Performance analysis of the IEEE 802.11 distributed coordination function," Selected Areas in Communications, IEEE Journal, vol. 18, pp. 535-547, 2000.

[12] R. Haywood et al., "Investigation of H.264 Video Streaming over an IEEE 802.11e EDCA Wireless Testbed," in Proc. IEEE International Conference on Communications, 2009, pp. 1-5.
[13] Y. P. Fallah et al., "Scheduled and contention access transmission of partitioned H.264 video over WLANs," in Proc. Global Telecommunications Conference, 2007. GLOBECOM '07. IEEE, 2007, pp. 2134-2139.

[14] H. Zen et al., "Adaptive segregation-based MAC protocol for realtime multimedia traffic in WLANs," in Proc. 15th IEEE International Conference on Network, 2007, pp. 461-466.

[15] W. Ping and Z. Weihua, "A token-based scheduling scheme for WLANs supporting voice/data traffic and its performance analysis," IEEE Transactions on Wireless Communications, vol. 7, pp. 17081718,2008

[16] P. Koutsakis, "Token and self policing-based scheduling for multimedia traffic transmission over WLANs," Vehicular Technology, IEEE Transactions on, vol. PP, pp. 1-1, 2011.

[17] D. Skordoulis et al., "Adaptive delayed channel access for IEEE 802.11n WLANs," in Proc. 2008. 4th IEEE International Conference on, 2008Circuits and Systems for Communications, pp. 167-171, 2008.

[18] E. N. Ciftcioglu and O. Gurbuz, "Opportunistic Scheduling with frame aggregation for next generation wireless LANs," in Proc. 2006. ICC '06. IEEE International Conference on Communications, 2006, pp. 5228-5233.

[19] F. Wang et al., "QoS based scheduling in the downlink of multi-user wireless systems (extended)," Computer Communications, vol. 32, pp. 1257-1262, 2009.

[20] T. Selvam and S. Srikanth, "A frame aggregation scheduler for IEEE 802.11n," in Proc. 2010 National Conference on Communications (NCC), 2010, pp. 1-5.

[21] B. Maqhat, M. D. Baba, and R. A. Rahman, "A-MSDU real time traffic scheduler for IEEE802.11n WLANs," in Proc. 2012 IEEE Symposium on Wireless Technology and Applications (ISWTA), 2012, pp. 286-290.

[22] A. P. Stephens et al., IEEE 802.11 Wireless LANs: Usage Models. Technical Report, IEEE 802.11n working document 802.1103/802r23, 2004

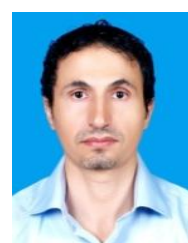

Bakeel Hussein received his B.Sc. degree in electrical engineering from Mutah University, Jordan in 2004 and his M.Sc. degree in telecommunication and information engineering from Universiti Teknologi MARA, Malaysia (UiTM), in 2008. Currently, he is working towards the Ph.D. degree at the Centre for Computer Engineering Studies, Faculty of Electrical Engineering, Universiti Teknologi MARA, UiTM.

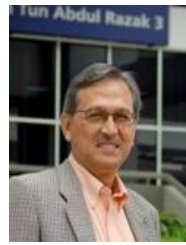

Mohd Dani Baba received his $\mathrm{PhD}$ degree from University of Sussex, UK in 1996. Currently he is a professor at the Centre for Computer Engineering Studies, Faculty of Electrical Engineering, Universiti Teknologi MARA. His research interests include computer networking, cloud computing, wireless networking, wireless sensor networks and mobile communication.

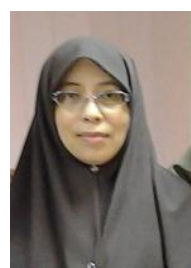

Ruhani Ab Rahman is a lecturer at Faculty of Electrical Engineering, Universiti Teknologi MARA. R.Ab Rahman received her degree in B.Eng in electrical and electronics engineering in 1988 from Portsmouth Polytechnic, UK and MSc in data networks and telecommunication from University of Salford, UK in 1998. She has been working with UiTM since 1988 and her major interests are networking, mobile networks and engineering education. R.Ab Rahman an active member in Cisco Malaysia Academy Council. She is also the member of IEEE.

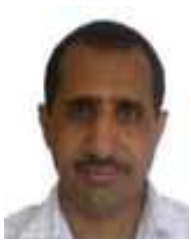

Anwar Saif received the B.S. degree in computer engineering from the Technical University of Budapest, Hungary, in 1996. He received his M.S. degree in networking and distributed computation from University Putra Malaysia, Malaysia (UPM), in 2008. And the PhD from University Putra Malaysia, Malaysia (UPM), in 2011.Currently, he is a lecturer at the Faculty of Electrical Engineering, Sana'a University. 